\title{
Influence of aerodynamic configuration of a streamline box girder on bridge flutter and vortex-induced vibration
}

\author{
Qi WANG* ${ }^{*}$ Haili LIAO, Mingshui LI, Cunming MA \\ Research Center for Wind Engineering, Southwest Jiaotong University, Chengdu 610031, China
}

\begin{abstract}
Streamline box girders are widely applied in the design and construction of long-span bridges all over the world. In order to study the influence of modifications of aerodynamic configuration and accessory components on flutter and vortex-induced vibration (VIV), more than 60 cases were tested through a 1:50 scale section model. The test results indicates that the aerodynamic configuration and accessory components of streamline box girders can significantly affect the wind-induced vibration of bridge, which is in good agreement with the experience of past researchers. From the tests carried out, it is observed that if the horizontal angle of the inclined web of the streamline box girder is below $16^{\circ}$, the critical flutter wind speed of bridge will increase remarkably, and the VIV will diminish. The test results also show that the $15^{\circ}$ inclined web can restrain the formation of vortex near the tail, and consequently improve the performance of aerodynamic stability of long-span bridges. Finally, a new streamline box girder with $15^{\circ}$ inclined web was presented and strongly recommended in the aerodynamic configuration design of long-span bridges.
\end{abstract}

Key words: streamline box girder; aerodynamic configuration; wind tunnel test; flutter; vortex-induced vibration

(C) 2011 JMT. All rights reserved.

\section{Introduction}

$\mathrm{S}$ ince its successful application to the Severn Bridge of Great Britain, the streamline box girder has been widely used in the design and construction of long-span bridges all over the world. Its aerodynamic configuration and accessory components have great impacts on bridge wind-induced vibration, especially the flutter and vortex-induced vibration (VIV). Larsen [1] conducted a series of wind tunnel tests on the girder of the Great Belt Bridge, and discussed the influence of railing, guide wing, and rostrum on the flutter. He found that the railings of low porosity weakened the aerodynamic stability, the blunt rostra decreased the flutter wind speed, and the guide wing strengthened the aerodynamic stability. Similar results were obtained by Miyata [2] and Bruno [3]. Wilde et al. [4] designed an active deck flaps control system to strengthen the aerodynamic stability of long-span bridges, but this system could increase the construction and maintenance cost of bridges, and its feasibility and efficiency in real-world applications still need to be validated. Yang and Ge [5] studied the effects of central stabilizer on aerodynamic control of long-span

Received Nov. 14, 2011; revision accepted Dec. 5, 2011

${ }^{*}$ Corresponding author. E-mail: wangchee_wind@swjtu.edu.cn (Q. WANG)

(C) 2011 JMT. All rights reserved

doi: 10.3969/j.issn.2095-087X.2011.04.007 bridges. The results indicated that the stabilizer did good to aerodynamic stability, and its fixed position must be confirmed by wind tunnel tests. However, the stabilizer did not fit for a traditional box girder. Through the wind tunnel tests and experiments [1-5], it was found that the flutter of long-span bridges could be avoided by optimizing the aerodynamic configuration and deck details. Compared with the studies on flutter of the streamline box girders of long-span bridges, however, the studies on VIV are not often seen. Larsen et al. [6] discussed the guide vane of box girder for suppression of the VIV of the Great Belt Bridge. He provided a general design of the guide vane for the twin box girder of Stonecutter Bridge in Hongkong [7], but this design would raise the construction cost. Diana et al. [8] investigated the vortex-shedding phenomena of the multi-box deck shape of Messina Strait Bridge and discovered the remarkable VIV phenomena in the bridge, but he did not provide the effective measures to suppress the VIV of the triplet girder. Ge et al. [9] carried out wind tunnel tests and field tests for the VIV of Xihoumen Bridge with a twin box girder, and found that the windbreak could control VIV effectively but would increase the drag force of girder and weaken the aerodynamic stability of the bridge. The aforementioned work indicates that there are lack of effective and practical measures to suppress VIV and maintain the aerodynamic stability of long-span bridges. 
In order to clarify the influence of accessory components and aerodynamic configuration on the aerodynamic stability and VIV of long-span bridges, this paper deals with wind tunnel tests through a 1:50 scale section model of streamline box girder. More than 60 cases of deck configuration were tested, such as the bridge railings with different porosities in the sideway, the position of the rail of bridge inspection car, the obliquity and width of guide wing, the edge configuration of the section (rostrum), and the inclination of the inclined web. Based on the tests, a new design for aerodynamic configuration of bridge girder is put forward, which could ensure the superior aerodynamic stability and less VIV of long-span bridges.

\section{Wind tunnel test model}

The design girder cross-section of the Nanjing 4th bridge, which crosses the Yangtze River and is located in Jiangsu Province of China, with the main span of $1418 \mathrm{~m}$, was selected to carry out wind tunnel tests. The bridge deck is originally designed a trapezoidal steel box girder with overall width of $37.7 \mathrm{~m}$ and a height of $3.4 \mathrm{~m}$, and the inclination of inclined web is $22^{\circ}$, as shown in Fig. 1. For long-span bridges, the aerodynamic stability is a governing factor in the design. According to the wind statistic data and the Wind-Resistant Design Specification for Highway Bridges [10], the flutter checking wind speed of the bridge is calculated as up to $60.8 \mathrm{~m} / \mathrm{s}$. However, the critical flutter wind speed of the original girder is found by intensive wind tunnel testing of the section model only $45 \mathrm{~m} / \mathrm{s}$. Apparently it falls short of the required flutter checking wind speed. Hence, in the aerodynamic design of this bridge, it is necessary to adopt some countermeasures to ensure the critical flutter wind speed meets the requirements of wind resistance design. And on the other hand, we attempt to find the necessary measures to suppress the VIV through the tests.

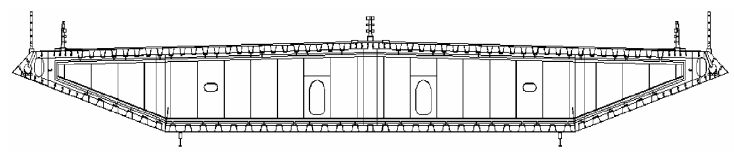

Fig. 1 Outline of the streamline box girder in the tests

The wind tunnel tests were conducted in the second test section of XNJD-1 wind tunnel. The model is $2.1 \mathrm{~m}$ long, and its width and height vary with the change of aerodynamic shape. The mass of the model is $23.52 \mathrm{~kg}$, and the mass moment of inertia is $1.407 \mathrm{~kg} \cdot \mathrm{m}^{2}$. The vertical and torsional frequencies of the test system are $1.76 \mathrm{~Hz}$ and $4.03 \mathrm{~Hz}$, respectively, and the wind speed ratio between model and real bridge is 3.28 . The damp- ing ratios of the test system in vertical and torsional directions are 0.45 and 0.48 , respectively. The aeroelastic section model in the wind tunnel tests is shown in Fig. 2.

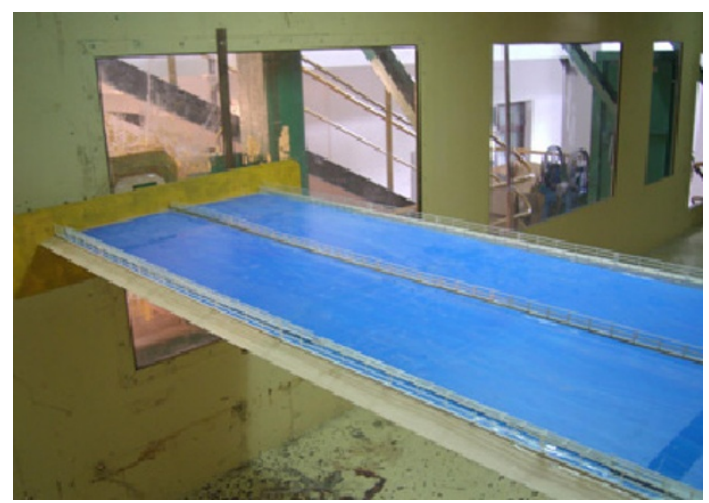

Fig. 2 Section model in the wind tunnel tests

\section{Influence of bridge railing}

The railings of two porosities $(90 \%$ and $60 \%)$ at three different locations on deck were tested under three attack angles $\left(-3^{\circ}, 0^{\circ}\right.$ and $\left.3^{\circ}\right)$. The three locations are as follows: one is on the edge (the original design), the other two are $5 \mathrm{~mm}$ (prototype $250 \mathrm{~mm}$ ) and $10 \mathrm{~mm}$ (prototype $500 \mathrm{~mm}$ ) away from the sideway edge. The test results are given in Table 1.

Table 1 Critical flutter wind speeds for different railings

\begin{tabular}{|c|c|c|c|c|}
\hline \multirow{2}{*}{ Porosity } & \multirow{2}{*}{ Position } & \multicolumn{3}{|c|}{ Attack angle } \\
\hline & & $-3^{\circ}$ & $0^{\circ}$ & $+3^{\circ}$ \\
\hline $90 \%$ & $\begin{array}{l}\text { In } \\
\text { pos }\end{array}$ & $>74.6$ & 73.0 & 61.3 \\
\hline $60 \%$ & & $>75.3$ & 57.6 & 44.8 \\
\hline $60 \%$ & $\begin{array}{r}250 \\
\text { in }\end{array}$ & $>74.8$ & 58.6 & 42.4 \\
\hline $60 \%$ & $\begin{array}{r}500 \\
\text { in }\end{array}$ & $>76.2$ & 57.9 & 44.0 \\
\hline
\end{tabular}

We find that the railings with high porosity strengthen the aerodynamic stability. Similar findings were also reported in Refs. [1-3]. The railings of low porosity mean the deck configuration being in the form of an H-shape, which may lead to flow separation. Then a rhythmic vortex shedding will lead to the generation of separation bubbles above the deck. The vortex creation and drift process will not only dramatically weaken the stability of girder, but also increase the amplitude of VIV [11-12]. The test results in Table 1 indicate that the 
railings of low porosity weaken the aerodynamic performance of bridge girder. However, the railings of high porosity are harmful to the safety and durability of longspan bridges. Therefore, in the design of railings, both the safety of bridge and the aerodynamic performance of the bridge girder should be ensured. Table 1 also shows that the critical wind speed of the girder is not sensitive to the railing position change.

\section{Influence of the rail of bridge inspection car}

The aerodynamic stability of the girder may be sensitive to the distance between the rail top and the girder bottom, called the rail gap, and also sensitive to the fixed position of rail on the girder. Tests on three gaps were carried out to investigate the potential influence. The gap was increased by $1 \mathrm{~mm}$ and $3 \mathrm{~mm}$ (prototype $5 \mathrm{~cm}$ and $15 \mathrm{~cm}$ ) in the tests (the porosity of railings is $90 \%$ ). Flutter critical speeds of these sections were measured, and are summarized in Table 2. It is found that increasing gap is beneficial to the critical wind speed at $0^{\circ}$ and $-3^{\circ}$ attack angle, but harmful to the aerodynamic stability of the girder at $+3^{\circ}$ attack angle. It can be seen from Table 2 that the critical flutter wind speed decreases with an increase in the rail gap. Thus, increasing the rail gap is not a good choice to strengthen the aerodynamic stability of the girder.

Table 2 Critical flutter wind speed under different gap

\begin{tabular}{|c|c|c|c|c|c|}
\hline \multirow{2}{*}{ Case } & \multirow{2}{*}{\multicolumn{2}{|c|}{ Distance }} & \multicolumn{3}{|c|}{ Attack angle } \\
\hline & & & $-3^{\circ}$ & $0^{\circ}$ & $+3^{\circ}$ \\
\hline $\begin{array}{l}\text { Initial } \\
\text { design }\end{array}$ & & $\begin{array}{l}5 \mathrm{~mm} \text { from } \\
\text { the bottom }\end{array}$ & $>79.0$ & 73 & 61.3 \\
\hline $\begin{array}{l}\text { Increment } \\
\text { of } 1 \mathrm{~mm}\end{array}$ & & $\begin{array}{l}6 \mathrm{~mm} \text { from } \\
\text { the bottom }\end{array}$ & $>81$ & $>80$ & 59.6 \\
\hline $\begin{array}{l}\text { Increment } \\
\text { of } 3 \mathrm{~mm}\end{array}$ & & $\begin{array}{l}8 \mathrm{~mm} \text { from } \\
\text { the bottom }\end{array}$ & $>84$ & $>82.5$ & 58.1 \\
\hline
\end{tabular}

The sketch maps for three different fixed positions of rails are shown in Fig. 3, and the VIV test results of the three cases under $+3^{\circ}$ attack angle are shown in Fig. 4 . If the rail was close to the corner of the girder bottom (see Fig. 3(a)), which is the separation point of incoming flow, the amplitude of VIV would become too large to exceed the code limit. If the rail was far from the corner of the girder bottom (see Fig. 3(b)), the amplitude of VIV would become small, or tend to zero. If the guide vane was fixed at both sides of the rail in the original design, shown in Fig. 3(c), the VIV amplitude could de- crease significantly. The position of rail has a large effect on the VIV.

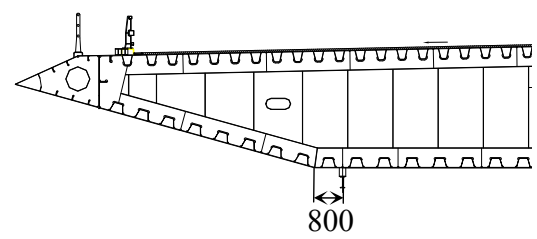

(a) Original design

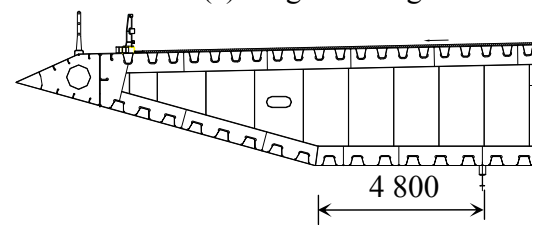

(b) The adjusted position of rail

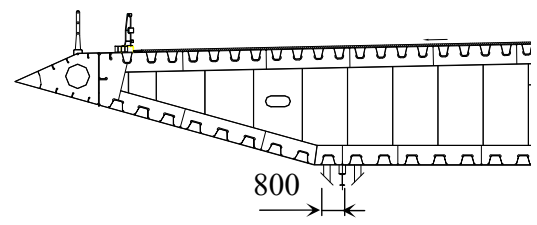

(c) Original design with guide vane

Fig. 3 Positions of the rail of bridge inspection car (unit: $\mathrm{mm}$ )

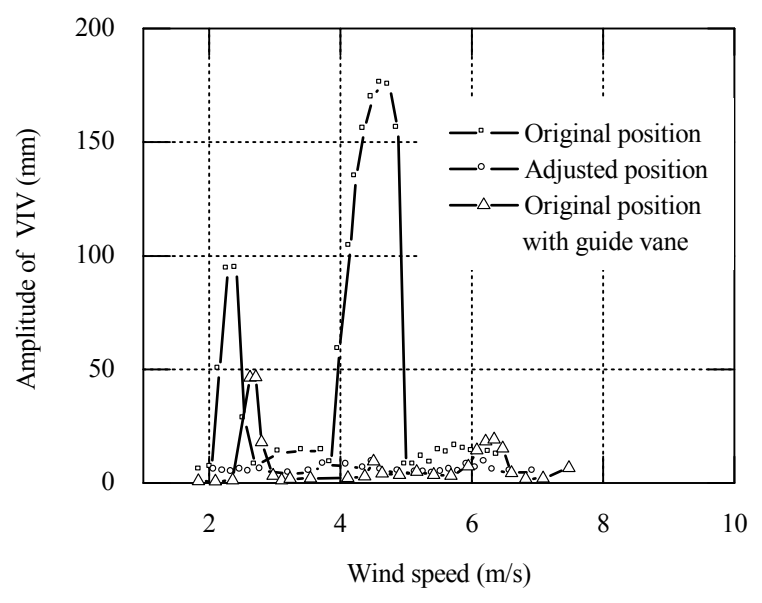

Fig. 4 VIV amplitudes for different rail positions

\section{Influence of guide wing}

The guide wing on the edge of sideway, shown in Fig. 5, can smoothen the airflow passing through the section, which may strengthen the aerodynamic stability [1-3]. A total of nine different types of guide wings were employed in the intensive wind tunnel tests, with different widths and obliquities. The railing porosity was $60 \%$ for the section model in the tests. Flutter wind speeds were obtained in the tests for the section model under attack angles of $+3^{\circ}$ and $0^{\circ}$, and the results are shown in Table 3 .

It is observed from Table 3 that, with an increase in the width of the guide wing with a positive obliquity, the aerodynamic stability is strengthened even though the 
railings have a low porosity. However, the guide wing will increase the complexity of the structure and its construction, particularly in the location of rostra, and the maintenance cost will increase accordingly. Therefore, a guide wing fixed at girder sides is not recommended in the design unless there is no alternative.

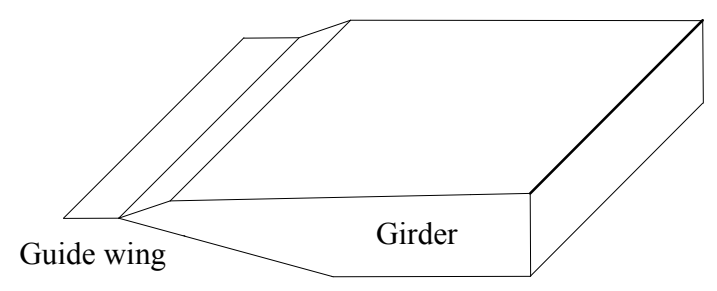

(a)

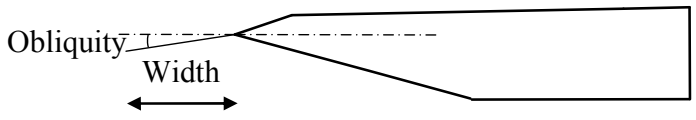

(b)

Fig. 5 Outline of guide wing

Table 3 Critical flutter wind speed with guide wings $(\mathrm{m} / \mathrm{s})$

\begin{tabular}{ccccc}
\hline \multicolumn{2}{c}{ Guide wing } & & \multicolumn{2}{c}{ Attack angle } \\
\cline { 1 - 2 } \cline { 5 - 5 } Width $(\mathrm{cm})$ & Obliquity $\left(^{\circ}\right)$ & & $0^{\circ}$ & $3^{\circ}$ \\
\hline 50 & +15 & & 52.2 & 44.9 \\
50 & 0 & 51.47 & 41.98 \\
50 & -42 & 54.75 & 42.34 \\
100 & +15 & 54.96 & 52.05 \\
100 & 0 & 52.78 & 50.23 \\
100 & -42 & 58.77 & 45.63 \\
125 & +15 & 60.23 & 63.88 \\
125 & 0 & 56.94 & 55.48 \\
125 & -42 & 62.78 & 49.64 \\
\hline
\end{tabular}

\section{Influence of rostrum}

The critical wind speed is sensitive to the shape of the rostrum [1-3,11]. Therefore, the rostrum with different widths and angles was taken into account in the tests. The angle of the rostrum varied from $57^{\circ}$ to $25^{\circ}$; correspondingly, the width varied from $1.9 \mathrm{~m}$ to $3.3 \mathrm{~m}$. A total of 21 model cases were tested. The results are shown in Table 4.

It is noted that with an increase in the rostrum width and a decrease in the rostrum angle, the critical flutter wind speed increases. However, the critical flutter wind speed declines when the width of rostrum exceeds $3 \mathrm{~m}$,
Table 4 Critical flutter wind speeds with different rostra

$(\mathrm{m} / \mathrm{s})$

\begin{tabular}{ccccc}
\hline \multicolumn{2}{c}{ Rostrum } & \multicolumn{4}{c}{ Attack angle } \\
\cline { 1 - 2 } Angle $\left(^{\circ}\right)$ & Width $(\mathrm{m})$ & $+3^{\circ}$ & $-3^{\circ}$ & $-3^{\circ}$ \\
\hline 57 & 1.9 & 51.5 & $>69.6$ & $>70.6$ \\
47 & 2.1 & 53.7 & $>70.7$ & $>70.3$ \\
41 & 2.3 & 54.6 & $>71.2$ & $>70.5$ \\
37 & 2.5 & 56.7 & $>70.8$ & $>71.2$ \\
33 & 2.7 & 58.6 & $>71.5$ & $>75.2$ \\
30 & 3.0 & 63.4 & $>72.5$ & $>70.9$ \\
25 & 3.3 & 59.3 & $>71.3$ & $>72.8$ \\
\hline
\end{tabular}

which may weaken the stability, and the reason will be discussed in the later section of this paper.

Similar conclusions were obtained from the VIV tests. The amplitude of the VIV decreases with a decrease in the angle of the rostrum (see Fig. 6). Thus, it can be inferred that the blunter the rostrum, the larger the VIV amplitude. The reason is that being the first separation point of the incoming flow, when the rostrum becomes blunter, the flow is easier to separate, and the vortex will form and drift over the girder, which can intensify the VIV amplitude. According to this result, a blunter rostrum, or the similar aerodynamic components which make the rostrum blunter, such as the ordinary sidewalk board, is not recommended in the design.

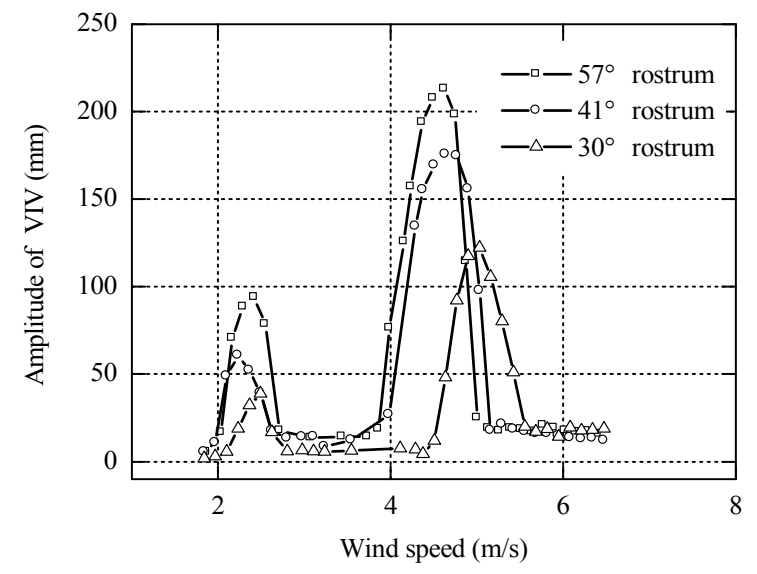

Fig. 6 VIV test results with different rostra

\section{Influence of inclined web}

A wide and acuminate rostrum is difficult to fabricate and fix, implying more cost in design and construction, although it can strengthen the aerodynamic stability of the girder. An alternate way is to decrease the steepness of sidewall slope to make the deck cross-section more 
streamlined. The critical flutter wind speed of the girder model with different slopes of the inclined web is shown in Table 5 (the width of rostrum was $2.4 \mathrm{~m}$ ), and the value increases with the decrease of the slope. When the slope is decreased to $15^{\circ}$, the critical flutter wind speed rose to $67.1 \mathrm{~m} / \mathrm{s}$, directly improving the flutter instability by $10 \%$. From Table 5 , we can obtain an ideal section of girder for improving flutter instability: short rostrum, no guide wing, and railings of low porosity.

Table 5 Critical flutter wind speed with different slopes

\begin{tabular}{cccc}
\hline \multirow{2}{*}{ Slope of inclined web $\left(^{\circ}\right)$} & \multicolumn{4}{c}{ Attack angle } \\
\cline { 2 - 4 } & $+3^{\circ}$ & $0^{\circ}$ & $-3^{\circ}$ \\
\hline 22 & 44.8 & 57.6 & $>75.3$ \\
20 & 56.7 & $>70.8$ & $>71.2$ \\
18 & 60.4 & $>72.5$ & $>70.9$ \\
15 & 67.1 & $>71.3$ & $>73.5$ \\
\hline
\end{tabular}

In addition, we have a new finding in the VIV tests. When the slope of inclined web is decreased to $15^{\circ}$, without the railings and the rail of the bridge inspection car, there is no VIV phenomenon. The results of VIV tests in different cases are shown in Fig. 7, where the slope of inclined web is $22^{\circ}$ for original design, $15^{\circ}$ for the adjustments without railings and without rail. Compared with the original design, the bare girder section without aerodynamic accessory components does not experience VIV. There is a clear conclusion that the rail of the inspection car on the bottom of girder will intensify VIV. The same conclusion was reached by Larsen [14] in 2008, who first discovered and documented this phenomenon. Furthermore, he verified this finding by considering two other bridges.

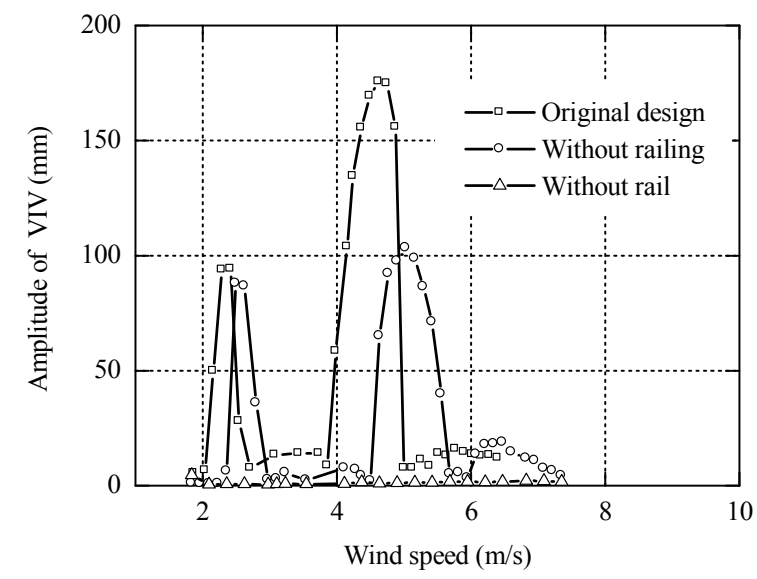

Fig. 7 Comparison between different VIV test results

\section{Discussion on aerodynamic performance improvement}

Based on the experience from vortex shedding tests, Larsen [13] found that the streamline box sections were similar to the airfoils at aerodynamic performance, and concluded that the flow along the bottom plate would stay mainly attached if the slope of inclined web was less than approximately $16^{\circ}$. He conducted a series of special tests and verified the above conclusion through the flow visualization technique [14]. He also gave an example about the design for a two span suspension bridge in Chile, whose inclined web angle was $14.8^{\circ}$, and no VIV was observed in the wind tunnel testing. On the contrary, explicit VIV was observed for the box girders of the Great Belt East Bridge and Osterøy Bridge, whose inclined web slope angles were $26.6^{\circ}$ and $29.5^{\circ}$, respectively [14].

By analyzing Larsen's test results and the test results of Fig. 7, we can draw the conclusion: vortex shedding excitation originated from rhythmic vortex formation under the downwind inclined web of the box section can be eliminated by choosing the slope of inclined web at about $15^{\circ}$ and shielding rail (see the test case without rail in Fig. 7).

Similar to a box section in the vortex shedding vibration status, in the flutter critical status, two vortices with opposite directions were observed at the both sides of the nose-tail line of Great Belt East Bridge section through the PIV (particle image velocimetry) technique [15]. The two vortices could give the girder enough momentum to increase its VIV amplitude in a short time and finally made the girder instable. When the wind speed was low and below the flutter critical speed, the positive vortex below the nose-tail line was more powerful than the above negative one, and the aerodynamic force was just a static lift force; when the wind speed increased close to the flutter critical speed, the negative vortex above the nose-tail line was strengthened to a level as powerful as the positive one below, and the aerodynamic force fluctuated [15]. If the frequency and the phase of the fluctuated force induced by vortex shedding are close to the bridge's modal frequency and phase, the flutter of girder will occur soon.

In the tests conducted in this paper, when the slope of the inclined web is decreased to $15^{\circ}$, there is a small dead wake region below the nose-tail line and the flow along the bottom plate stays mainly attached to the web, which makes it more difficult for formation of a large vortex. When the wind speed increases and approaches to the original critical flutter wind speed $(45 \mathrm{~m} / \mathrm{s})$, the opposite vortices can not become powerful, and can not give the girder powerful and efficient excitation. Fig. 8 shows the two vortices near the tail of the girder at this 
status. They are so asymmetrical that they can not produce the fluctuated aerodynamic force, and thus, the bridge remains stable. When the wind speed continues to increase, the equivalent opposite vortices will form at the similar position and the rhythmic excitation will return, which introduce aerodynamic instability to the girder once more. Fig. 9 reflects the status of the vortex moment at the wind speed approaching to the flutter critical value, and the equivalent vortices near the tail of the girder can produce the fluctuated aerodynamic force that makes the bridge instable.

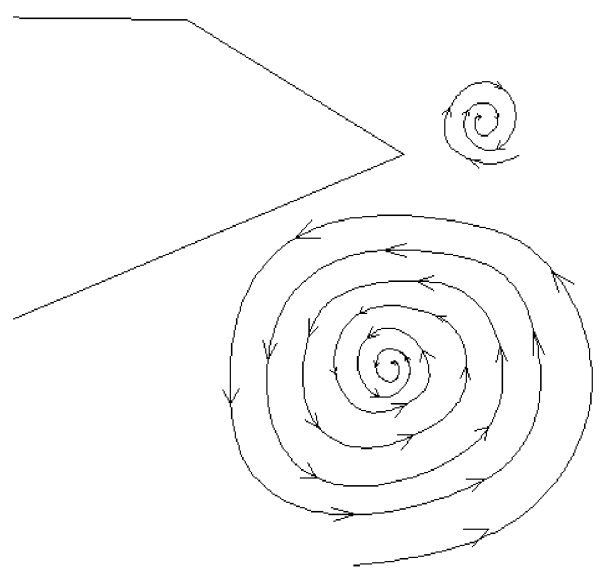

Fig. 8 Vortex moment at the low wind speed (below the critical value)

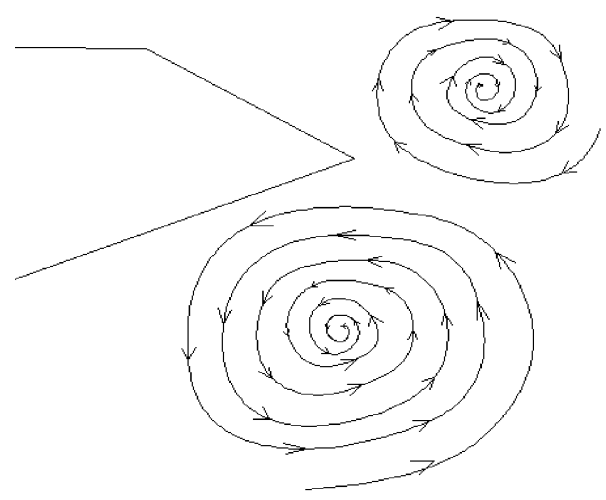

Fig. 9 Vortex moment when the wind speed approaches to the critical value

Therefore, vortex shedding excitation originated from the equivalent opposite vortices near the tail of the box section can be restrained and delayed by choosing the inclined web angle at $15^{\circ}$. The test results in Table 5 can verify this conclusion. In aerodynamic stability design of long-span bridges, to increase the critical flutter wind speed, the same design of the bridge girder with the inclined web at $15^{\circ}$ is recommended.

This explanation can also be extended to interpret the results shown in Table 4 . When the slope of the inclined web is less than $16^{\circ}$, the increase of the width of the rostrum leads to the formation of a large vortex, which is a disadvantage to the girder stability and make the critical flutter wind speed decrease. Table 6 shows the test results of the critical flutter wind speed with different width of rostra for inclined web of $15^{\circ}$. We can conclude that the short rostra are of benefit to the girder stability when the slope of inclined web is $15^{\circ}$.

Table 6 Critical flutter wind speed (slope $15^{\circ}$ )

$(\mathrm{m} / \mathrm{s})$

\begin{tabular}{cccccc}
\hline \multicolumn{2}{c}{ Rostra } & \multicolumn{4}{c}{ Attack angle } \\
\cline { 5 - 6 } Width $(\mathrm{m})$ & Angle $\left(^{\circ}\right)$ & $+3^{\circ}$ & $0^{\circ}$ & $-3^{\circ}$ \\
\hline 2.4 & 40 & 67.1 & $>71.3$ & $>73.5$ \\
2.6 & 36 & 62.0 & $>70.8$ & $>72.7$ \\
2.8 & 33 & 61.1 & $>71.5$ & $>72.4$. \\
\hline
\end{tabular}

It should be noted that the explanation documented above is based on the Larsen's research [14] on the vortex shedding vibration of streamlined box girder, and Zhang's research [15] on the flutter critical status of Great Belt East Bridge by PIV technique. The explanation is obtained by a logical deduction about aerodynamic stability improvement. It needs to be verified by the further study of the wind tunnel tests using PIV technique and computational fluid dynamics method in the future.

\section{Conclusions}

In this paper, more than 60 cases were tested through a 1:50 scale section model to study the influence of aerodynamic configuration and accessory components on the flutter and vortex-induced vibration (VIV), such as the railing, the rail of inspection car, the rostrum, the guide wing, and the slope of inclined web. From the tests, we can obtain several important conclusions for the aerodynamic design of streamline box girder in long-span bridges. The girders with railings of high porosity have higher critical flutter wind speed than the ones with railings of low porosity. The wide and acutance rostrum can strengthen the aerodynamic stability of the girder, but the width of rostrum cannot exceed $3 \mathrm{~m}$. The rail of the bridge inspection car fixed at the corner of bottom plate of box girder can intensify VIV. It is recommended that the rail should to be shielded by guide vanes.

If the inclined web with a $15^{\circ}$ angle is employed in the section design of long-span bridges, the flutter performance can be enhanced or the critical flutter wind speed can be increased dramatically. The VIV of longspan bridges under the practical structural damping can be also suppressed by this same design.

Through the wind tunnel tests, we find that the final design section of the Nanjing 4th bridge, including the 
railings of $60 \%$ porosity, shielded inspection car rail, inclined web with a slope of $15^{\circ}$ and $2.4 \mathrm{~m}$ wide rostrum, has a good aerodynamic stability, and the critical flutter wind speed is up to $67.1 \mathrm{~m} / \mathrm{s}$. Of course, there is no vortex shedding vibration observed in the 1:50 and 1:20 section model tests. This section model offers a good demonstration for the aerodynamic configuration designs of other long-span bridges. The points of this paper have been validated by the aerodynamic configuration design of Huangyi Bridge crossing the Yangtze River, which is located in Luzhou City, Sichuan Province.

\section{References}

[1] A. Larsen, Aerodynamic aspects of the final design of the $1624 \mathrm{~m}$ suspension bridge across the Great Belt, Journal of Wind Engineering and Industrial Aerodynamics, 1993, 48(2-3): 261-285.

[2] T. Miyata, Historical view of long-span bridge aerodynamics, Journal of Wind Engineering and Industrial Aerodynamics, 2003, 91(12-15): 1393-1410.

[3] L. Bruno, G. Mancini, Importance of deck details in bridge aerodynamics, Structural Engineering International, 2002, 12(4): 289-294.

[4] K. Wilde, P. Omenzetter, Y. Fujino, Suppression of bridge flutter by active deck-flaps control system, Journal of Engineering Mechanics, 2001, 127(1): 80-89.

[5] Y.X. Yang, Y.J. Ge, Some practices on aerodynamic flutter control for long-span cable supported bridges, In: Proceedings of the 4th International Conference on $\mathrm{Ad}$ vances in Wind and Structures (AWAS'08), Jeju, Korea, May 28-30, 2008.

[6] A. Larsen, S. Esdahl, J.E. Andersen, et al., Storebaelt suspension bridge-vortex shedding excitation and mitigation by guide vanes, Journal of Wind Engineering and Industrial Aerodynamics, 2000, 88(2-3): 283-296.
[7] A. Larsen, M. Savage, A. Lafrenière, et al., Investigation of vortex response of a twin box bridge section at high and low Reynolds numbers, Journal of Wind Engineering and Industrial Aerodynamics, 2008, 96(6-7): 934944.

[8] G. Diana, F. Resta, M. Belloli, et al., On the vortex shedding forcing on suspension bridge deck, Journal of Wind Engineering and Industrial Aerodynamics, 2006, 94(5): 341-363.

[9] Y.J. Ge, Y.X. Yang, F.C. Cao, VIV sectional model testing and field measurement of xihoumen suspension bridge with twin box girder, http://www.dist.unina.it/proc/2011/ICWE13/start/papers/502_8page_viv_se ctional_model_testing_and_field_measurement_of_xihoumen_suspension_bridge_with_twin_box_girder.pdf, 2011-10-30.

[10] JTGT D60-01-2004, Wind-Resistant Design Specification for Highway Bridges.

[11] J.Z. Song, Z.X. Lin, J.Y. Xu, Research and appliance of aerodynamic measure s about wind resistance of bridges, Journal of Tongji University (Natural Science), 2002, 30(5): 618-621 (in Chinese).

[12] C.J. Liu, Z.S. Guo, L.D. Zhu, Influence of railing curbstone structure on flutter stability of box main girder, Bridge Construction, 2008(2): 20-22,44 (in Chinese).

[13] A. Larsen, Aerodynamic stability and vortex shedding excitation of suspension bridges, In: Proceedings of the Fourth International. Conference on Advances in Wind and Structures (AWAS'08), Jeju, Korea, 2008.

[14] A. Larsen, A. Wall, Shaping of bridge box girders to avoid vortex shedding response, http://www.dist.unina.it/ proc/2011/ICWE13/start/papers/633_8page_shaping_of_ bridge_box_girders_to_avoid_vortex_shedding_response. pdf, 2011-10-28.

[15] W. Zhang, Y.J. GE, Flow field mechanism of wind induced vibration response of large span bridge influenced by guide vanes, China Journal of Highway and Transport, 2009, 22(3): 52-57 (in Chinese).

(Editor: Dongju CHEN) 